\title{
Short communication: Glucose and fructose concentrations and expression of glucose transporters in 4- to 6-week pregnancies collected from Holstein cows that were either lactating or not lactating
}

\author{
M. C. Lucy, ${ }^{1}$ J. C. Green, J. P. Meyer, A. M. Williams, E. M. Newsom, and D. H. Keisler \\ Division of Animal Sciences, University of Missouri, Columbia 65211
}

\begin{abstract}
Glucose is an essential nutrient for the conceptus. The objective was to determine if lactation affected the amount of glucose crossing the placenta by measuring glucose and fructose in placental fluids in lactating and nonlactating cows. Holstein cows were assigned to one of 2 treatments immediately after parturition [lactating $(\mathrm{n}=23)$ or nonlactating (dried off immediately after calving; $\mathrm{n}=20$ )]. Pregnant cows were slaughtered at one of $3 \mathrm{~d}$ of pregnancy (d 28, 35, or 42) and tissues were collected. Plasma glucose and insulin were less in lactating cows. Pregnancies collected from lactating cows had less glucose and fructose in placental fluids compared with those from nonlactating cows. Relative to endometrium, the placenta expressed greater amounts of the glucose transporters SLC2A1 (Glut1), SLC2A3 (Glut3) and SLC2A4 (Glut4) mRNA. The mRNA for $S L C 2 A 1$ decreased whereas the mRNA for SLC2A4 increased from d 28 to d 42 of pregnancy. Stepwise regression analyses for fetal and placental weight (dependent variable) retained day of pregnancy and maternal plasma insulin concentrations in the final model. The conclusion is that lower blood glucose and insulin in lactating cows may lead to less glucose crossing the placenta and slower fetal development during lactation. The slower fetal development may predispose lactating cows to fetal loss if developmental milestones are not reached.
\end{abstract}

Key words: glucose, glucose transporter, dairy cow, pregnancy

\section{Short Communication}

Embryonic and fetal loss in dairy cattle occurs primarily during the first 6 to 8 wk of pregnancy (Diskin and Morris 2008). An initial period of loss occurs be-

Received February 21, 2012.

Accepted May 12, 2012.

${ }^{1}$ Corresponding author: lucym@missouri.edu tween d 14 and 20 when the filamentous embryo secretes interferon- $\tau$ for maternal recognition of pregnancy (Walsh et al., 2011). Following this initial period of loss, a second period of fetal loss ensues and lasts until approximately wk 8 of pregnancy (Santos et al., 2004). This second period coincides with the attachment of the placenta to the uterine wall via the formation of placentomes (King et al., 1979, 1980). The second period of loss is particularly frustrating for dairy farmers because a cow may be initially diagnosed pregnant and then later found to be not pregnant because the pregnancy has died between wk 4 and 8 (Whitlock and Maxwell, 2008). Given the importance of pregnancy to the economic viability of the dairy (DeVries, 2006), there is a major focus on understanding the factors that contribute to pregnancy loss so that it can be alleviated or eradicated on dairy farms.

A defining characteristic of the early postpartum dairy cow is her capacity to undergo nutrient partitioning to support the production of milk (Bauman and Currie, 1980). High-producing dairy cows have low blood concentrations of glucose because glucose is readily sequestered by the mammary gland for energy and the production of lactose (Lucy, 2004). The lactation-dependent depression in blood glucose results in low maternal blood insulin concentrations. The partitioning of glucose during lactation can potentially affect the pregnancy because glucose is the primary metabolic fuel for the developing conceptus (Battaglia and Meschia, 1978). Glucose crosses the uterus and placenta by facilitative transport (down its concentration gradient). The primarily glucose transporters in uterus and placenta are the facilitative transporters SLC2A1 (Glut1) and SLC2A3 (Glut3) (Hay, 2006; Frolova and Moley, 2011). Low circulating concentrations of glucose in maternal blood may lead to low glucose reaching fetal and placental tissues because glucose moves down its concentration gradient from maternal blood to the developing pregnancy. Fetal loss during early pregnancy might be explained, in part, by insufficient glucose reaching the pregnancy and a deficiency in metabolic fuel during critical windows of conceptus development. 
We hypothesized that lactation would negatively affect the rate of fetal development through a mechanism involving maternal glucose concentrations. To examine this hypothesis, we designed an experiment in which postpartum cows were assigned to 1 of 2 treatments immediately after parturition [lactating or nonlactating (dried off immediately after calving)] and had their pregnancies collected on d 28, 35, or 42 of gestation. The effects of lactation on the metabolic profiles of the cattle and the pregnancy itself were reported previously (Green et al., 2012). Fetal weight, placental weight, and placental fluid volume were greater in nonlactating compared with lactating cows (Green et al., 2012). In this short communication, glucose and fructose in the placental fluid, concentrations of insulin and glucose in the cows' plasma immediately preceding slaughter, and mRNA for glucose transporters in the uterus and placenta are reported.

A detailed description of the experiment has been reported (Green et al., 2012). Pregnant Holstein heifers $(\mathrm{n}=43)$ were randomly assigned to 1 of 2 treatment groups, either lactating $(\mathrm{n}=23)$ or not lactating (n $=20$ ). The nonlactating group was not milked after calving (i.e., dried off immediately, never milked). Both groups were housed in the same pen and fed the same diet that was formulated for lactating cows. All cows were inseminated from a single ejaculate of a high-fertility sire following a timed AI program. Cows that did not conceive to first insemination were subsequently reinseminated $12 \mathrm{~h}$ after an observed return to estrus or after a timed AI. Pregnant cows were slaughtered at 1 of 3 times of pregnancy: 28 d (lactating, $\mathrm{n}=6$; nonlactating, $\mathrm{n}=5$ ), $35 \mathrm{~d}$ (lactating, $\mathrm{n}=8$; nonlactating, $\mathrm{n}=$ 6 ), or 42 d (lactating, $\mathrm{n}=8$; nonlactating, $\mathrm{n}=6$ ). Four cows were not included in the analyses because they did not have a viable pregnancy (dead fetus found) at the time of slaughter (lactating, $\mathrm{n}=1$; nonlactating, $\mathrm{n}=1$ ), the volume of fetal fluid was too small for the analyses (nonlactating, $\mathrm{n}=1$ ), or they failed to become pregnant (nonlactating, $\mathrm{n}=1$ ).

Blood samples were collected from cows thrice weekly (Monday, Wednesday, and Friday mornings, approximately $2 \mathrm{~h}$ after feeding). For the purpose of this study, data for the plasma collected $1 \mathrm{wk}$ before slaughter are reported. A validated radioimmunoassay was used to determine the plasma concentrations of insulin (Kolath et al., 2006; intraassay CV $=6.9 \%$ ). Glucose (Pointe Scientific Inc., Canton, MI) and fructose (Biovision, Mountain View, CA) concentrations in plasma and placental fluids were measured by using colorimetric assay kits and by following the manufacturers' protocols.

Slaughter was done immediately after the morning milking. The reproductive tracts and a liver sample were collected, placed on crushed ice, and taken to the laboratory. The pregnancy (placental membranes encasing placental fluids and fetuses) was removed from the uterus. The placental fluids (mixture of both amniotic and allantoic fluids) were collected and measured, and a sample was frozen at $-80^{\circ} \mathrm{C}$. Samples of placenta, caruncular endometrium, and intercaruncular endometrium were frozen in liquid nitrogen so that they could be used for RNA analysis. For endometrial tissue collection (caruncular and intercaruncular endometrium), 5 to 10 representative $1 \times 1 \mathrm{~cm}$ samples were collected from each region, frozen in liquid nitrogen, and stored at $-80^{\circ} \mathrm{C}$. The 5 to 10 individual tissue samples within each tissue type from each cow were later combined and ground under liquid nitrogen with a mortar and pestle. Total cellular RNA was isolated by using the TRIzol reagent (Invitrogen, Carlsbad, CA). Caruncular endometrium and intercaruncular endometrium samples were further purified by using the QIAmp Mini Spin Column (Qiagen, Valencia, CA). The integrity of RNA was determined by visual inspection of $28 \mathrm{~S}$ and 18S RNA bands after agarose gel electrophoresis and by calculating the ratio of absorbance at $260 \mathrm{~nm}$ and $280 \mathrm{~nm}$. The RNA that was used had intact $28 \mathrm{~S}$ and 18S RNA bands and the 260:280 $\mathrm{nm}$ ratio was greater than 1.8. The RNA was diluted in water and stored at $-80^{\circ} \mathrm{C}$.

Total RNA was transcribed into cDNA by using the High Capacity cDNA Reverse Transcription Kit (Applied Biosystems, Foster City, CA). Quantitative reverse transcription (RT)-PCR was used to assay for the amount of cDNA (i.e., mRNA) in each sample. The reaction was performed in an ABI Prism 7700 machine (Applied Biosystems) using the QuantiTect SYBR Green PCR Kit (Qiagen) and primers specific for PPIA (cyclophilin A; housekeeping gene), SLC2A1, SLC2A3, and SLC2A4 (Supplementary Table 1; available online: http://www.journalofdairyscience.org/). The PCR products produced from the RT-PCR were DNA sequenced to verify amplification of the target sequence. Within each plate were high, medium, and low control samples (sequential 1:4 dilutions) of pooled cDNA that were run in triplicate. Each 96-well PCR plate contained a no- template control (water substituted for cDNA in the reaction) to ensure that no amplification occurred in samples without cDNA. Data from the serial dilutions of the control sample were used to calculate the amplification efficiencies for the RT-PCR (Supplementary Table 1; http://www.journalofdairyscience.org/) by using procedures described previously (Rhoads et al., 2008). Fold change differences for the respective samples were calculated relative to an internal control standard within each plate according to the procedures described previously (Rhoads et al., 2008). Gene expression was initially assayed by using a single 
RT-PCR plate that included all of the cDNA samples in duplicate from a single tissue (e.g., liver or placenta). Samples were then analyzed to compare the expression of target genes across different tissues.

Cows were assigned to a first-insemination pregnancy status based on whether they conceived or failed to conceive to first insemination. Plasma glucose concentration, plasma insulin concentration, placental fluid glucose concentration, total placental fluid glucose (concentration multiplied by fluid volume), placental fluid fructose concentration, and total placental fluid fructose were analyzed by using PROC GLM of SAS (SAS Institute Inc., Cary, NC). The statistical model included the effects of treatment (lactating versus nonlactating), status (pregnant versus nonpregnant) day (d 28, 35, 42), and all interactions of these effects. The amount of mRNA for SLC2A1,SLC2A3, and SLC2A4 was analyzed for each tissue independently by using the model described in the preceding sentence. In the samples that were used to directly compare tissues for SLC2A1, SLC2A3, and SLC2A4, the model included treatment, tissue, day, and interactions. A stepwise regression was performed with PROC REG of SAS. The dependent variable was fetal plus placental weight. The independent variables were day of pregnancy $(28,35$, or 42), plasma insulin concentrations, plasma glucose concentration, placental fluid glucose concentration, and placental fluid fructose concentration. Means are reported as least squares means \pm SEM. A result was viewed as statistically significant if $P<0.05$.

We measured plasma glucose and insulin concentrations in plasma during the week before slaughter to assess the temporal relationship between maternal insulin and glucose and the concentration of glucose in fetal fluids. Lactating cows had lower plasma glucose concentrations $(P<0.001)$ and lower placental fluid glucose concentration $(P<0.001)$ compared with nonlactating cows $(64.8 \pm 1.3$ and $73.1 \pm 1.3 \mathrm{mg} /$ $\mathrm{dL}$, respectively, for plasma and $20.4 \pm 1.6$ and 29.2 $\pm 1.7 \mathrm{mg} / \mathrm{dL}$, respectively, for placental fluids; Figure 1). Plasma insulin concentrations were also lower in lactating compared with nonlactating cows $(P<0.001$; $7.6 \pm 0.52$ and $11.2 \pm 0.5 \mathrm{ng} / \mathrm{mL}$, respectively). Cows that conceived after first insemination $(27 / 39 ; 69 \%)$ had greater placental fluid glucose concentrations than cows that conceived after second or third insemination $(12 / 39 ; 31 \%)(27.4 \pm 41.3$ and $22.2 \pm 2.0 \mathrm{mg} / \mathrm{dL} ; P$ $<0.05)$. We observed an effect of day of pregnancy on concentration of glucose in placental fluids because glucose decreased on d 42 of pregnancy $(27.6 \pm 2.2$, $27.7 \pm 2.2$, and $19.0 \pm 1.8 \mathrm{mg} / \mathrm{dL}$ on $\mathrm{d} 28,35$, and 42 , respectively; $P<0.005$; Figure $1 \mathrm{~B})$. The placental fluid fructose concentration $(5.5 \pm 0.5 \mathrm{mM})$ was not affected by treatment (lactating versus nonlactating),
A)

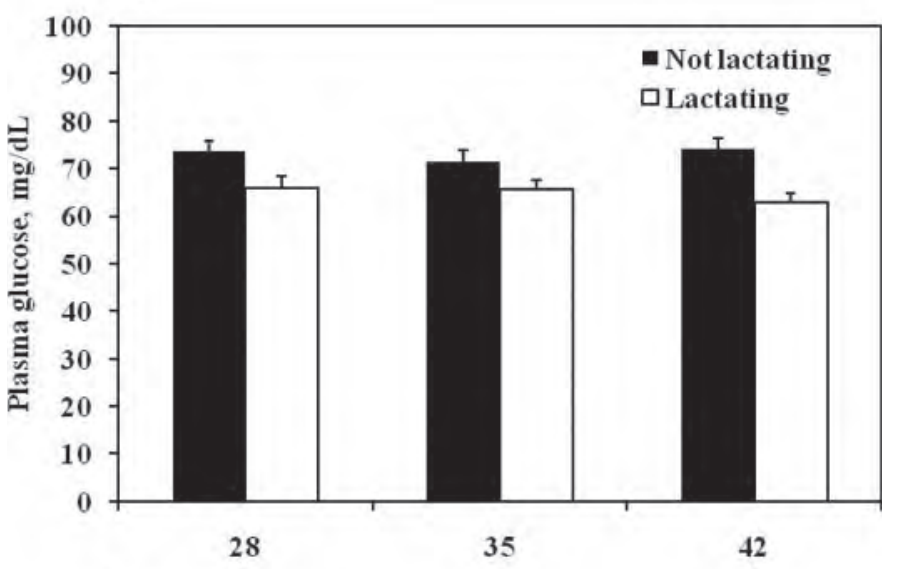

B)

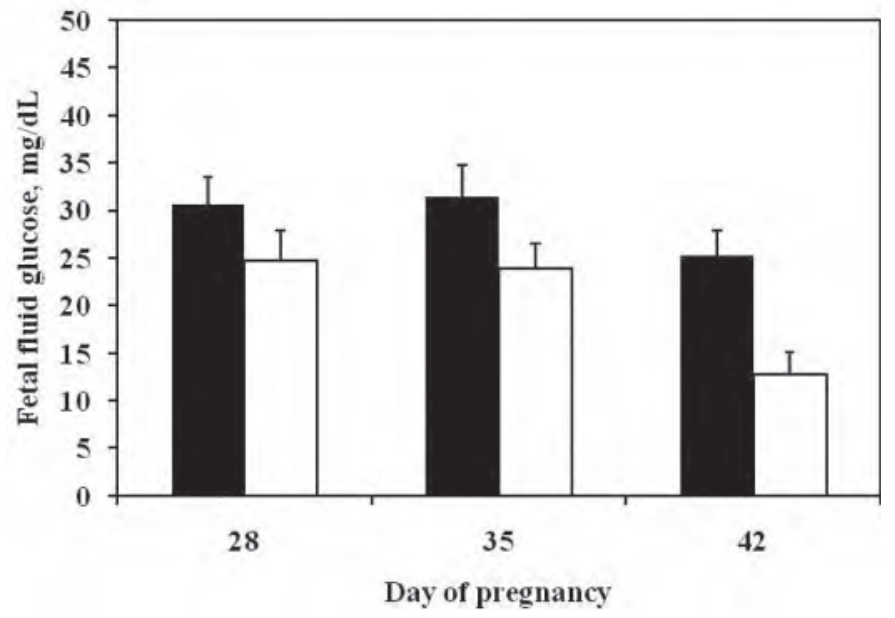

Figure 1. Least squares means ( \pm SEM, bars) for the maternal plasma concentration of glucose (A) and placental fluid concentration of glucose (amniotic and allantoic fluids combined, B) in Holstein cows that were either lactating or not lactating (dried off immediately after calving) and had tissues collected and sampled on d 28,35 , or 42 of pregnancy.

pregnancy status (pregnant versus nonpregnant), or day of pregnancy.

The total amount of glucose and fructose (volume of placental fluids multiplied by concentration) increased with increasing day of pregnancy $(P<0.001$ for glucose and fructose). We observed an effect of treatment for total placental fluid glucose $(P<0.01)$ and total placental fluid fructose $(P<0.01)$ : nonlactating cows had greater total amounts of glucose $(0.03 \pm 0.02,0.15 \pm$ 0.03 , and $0.22 \pm 0.02 \mathrm{~mol}$ vs. $0.02 \pm 0.03,0.08 \pm 0.02$, and $0.09 \pm 0.02 \mathrm{~mol}$; nonlactating versus lactating on $\mathrm{d}$ 28,35 , and 42 , respectively) and fructose $(0.10 \pm 0.11$, $0.53 \pm 0.12$, and $0.88 \pm 0.10 \mathrm{~mol}$ vs. $0.00 \pm 0.12,0.25 \pm$ 0.10 , and $0.51 \pm 0.11 \mathrm{~mol}$; nonlactating versus lactating on d 28,35 , and 42 , respectively). A treatment by day 


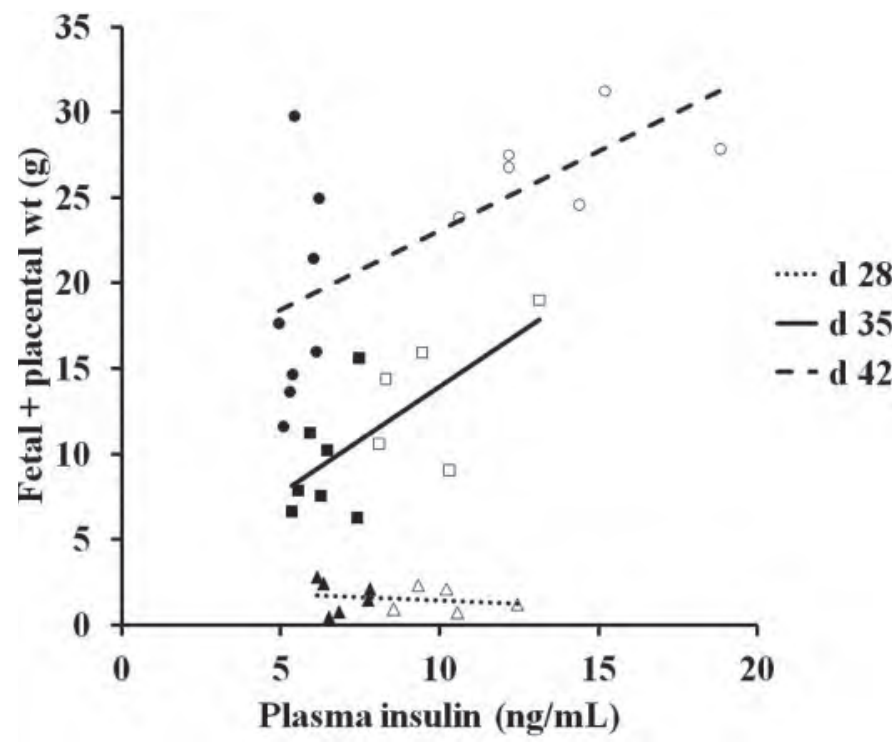

Figure 2. Linear regressions of fetal and placental weight on maternal plasma insulin concentrations for Holstein cows that were either lactating (solid symbols) or nonlactating (open symbols) and had their pregnancies collected on d 28 (triangles and dotted lines), d 35 (boxes and solid lines), or d 42 (circles and dashed lines). The linear regression for fetal and placental weight on maternal plasma insulin was significant on d 35 (weight $=1.25 \times$ insulin $\left.+1.5 ; \mathrm{R}^{2}=0.47 ; P<0.01\right)$ and $\mathrm{d} 42$ (weight $=0.93 \times$ insulin $\left.+13.8 ; \mathrm{R}^{2}=0.45 ; P<0.01\right)$ but not on $\mathrm{d} 28$ (weight $=-0.08 \times$ insulin $+2.2 ; \mathrm{R}^{2}=0.04$ ).

interaction $(P<0.05)$ for total glucose was detected but the treatment by day interaction for fructose was not significant.

When a stepwise regression was performed for fetal plus placental weight, the 2 effects that remained in the model were day of pregnancy $(P<0.001)$ and maternal plasma insulin concentrations $(P<0.01)$. When a linear model with day (discrete variable) and day by plasma insulin (continuous variable) was fit, the interaction of day by plasma insulin was significant $(P<0.01)$. Total fetal and placenta weight increased with increasing maternal plasma insulin for pregnancies collected on d 35
$(P<0.01)$ and $42(P<0.01)$ but did not increase for pregnancies collected on d 28 (Figure 2).

The placenta had greater SLC2A1 and SLC2A3 expression than liver, caruncular endometrium, or intercaruncular endometrium $(P<0.05$; Table 1$)$. The liver had greater SLC2A4 than placenta (approximately 3 -fold greater expression; $P<0.05$ ). Placenta had more SLC2A4 than either caruncular or intercaruncular endometrium $(P<0.05)$. Within liver, we found no effect of treatment, day, status, or interactions on the expression of PPIA, SLC2A1, or SLC2A3. We did observe an effect of treatment $(P<0.05)$ and day $(P<$ $0.05)$ on $S L C 2 A 4$ in liver. Lactating cows had less liver $S L C 2 A 4$ mRNA than nonlactating cows $(0.83 \pm 0.12$ and $1.19 \pm 0.11$, respectively; $P<0.05$ ). Cows on $d 28$ of pregnancy had less liver SLC2A4 than cows on d 35 or 42 of pregnancy $(0.69 \pm 0.14,1.20 \pm 0.17,1.15 \pm$ 0.12 , for d 28, 35, and 42, respectively). For caruncular endometrium, the amount of PPIA, SLC2A1, SLC2A3, and $S L C 2 A 4$ was not affected by treatment, day, status, or interactions. In intercaruncular endometrium, we observed an effect of day on PPIA expression $(P<$ $0.05 ; 0.32 \pm 0.07,0.55 \pm 0.09$, and $0.60 \pm 0.06$ for $\mathrm{d} 28$, 35 , and 42 , respectively) but the effects of treatment, day, and status were not significant for intercaruncular endometrial SLC2A1, SLC2A3, or SLA2A4. For placenta, the effect of day was significant for PPIA ( $P$ $<0.001)$, SLC2A1 $(P<0.001)$, and SLC2A4 $(P<$ $0.01)$. The placental expression of PPIA and SLC2A1 decreased from d 28 to 42 of pregnancy (Figure 3). The placental expression of $S L C 2 A 4$ was opposite to that of PPIA and SLC2A1 because SLC2A4 expression increased from d 28 to 42 of pregnancy. The effect of day was not significant for placental SLC2A3, and none of the other effects in the model (treatment, status, or interactions) were significant for placental PPIA, SLC2A1, SLC2A3, or SLC2A4.

We used an experimental design that included lactating and nonlactating cows so that we could test the ef-

Table 1. Least squares means $( \pm$ SEM) for the amount of mRNA (assessed by quantitative, reverse transcription-PCR) for glucose transporter genes SLC2A1,SLC2A3, and SLC2A4 in liver, placenta, caruncular endometrium, and intercaruncular endometrium ${ }^{1}$

\begin{tabular}{|c|c|c|c|c|}
\hline \multirow[b]{2}{*}{ Gene } & \multicolumn{4}{|c|}{ Tissue $^{1}$} \\
\hline & Liver & Placenta & $\begin{array}{l}\text { Caruncular } \\
\text { endometrium }\end{array}$ & $\begin{array}{l}\text { Intercaruncular } \\
\text { endometrium }\end{array}$ \\
\hline$\overline{S L C 2 A 1 \text { (Glut 1) }}$ & $0.11 \pm 0.41^{\mathrm{b}}$ & $8.97 \pm 0.41^{\mathrm{a}}$ & $0.001 \pm 0.41^{\mathrm{b}}$ & $0.001 \pm 0.41^{\mathrm{b}}$ \\
\hline SLC2A3 (Glut 3) & $0.02 \pm 0.74^{\mathrm{b}}$ & $13.57 \pm 0.74^{\mathrm{a}}$ & $0.001 \pm 0.74^{\mathrm{b}}$ & $0.001 \pm 0.74^{\mathrm{b}}$ \\
\hline$S_{L C 2 A 4}$ (Glut 4) & $25.39 \pm 1.67^{\mathrm{a}}$ & $8.39 \pm 1.67^{\mathrm{b}}$ & $0.03 \pm 1.67^{\mathrm{c}}$ & $0.03 \pm 1.67^{\mathrm{c}}$ \\
\hline
\end{tabular}

${ }^{\mathrm{a}-\mathrm{c}}$ Means within a row with different superscripts differ at $P<0.05$ (Duncan's multiple range test).

${ }^{1}$ From Holstein cows that were either lactating or nonlactating (dried off immediately after calving) and had tissues collected on d 28,35 , or 42 of pregnancy. 


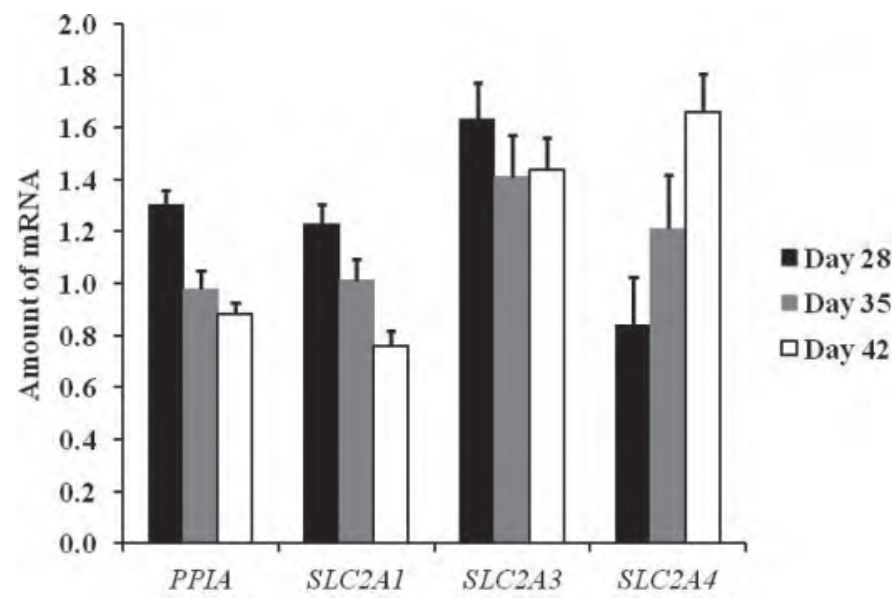

Figure 3. Least squares means $( \pm \mathrm{SEM})$ for the amount of placental mRNA for PPIA (cyclophilin A), SLC2A1 (Glut 1), SLC2A3 (Glut 3 ), and $S L C 2 A 4$ (Glut 4) on d 28, 35, or 42 of pregnancy. The data for Holstein cows that were either lactating or nonlactating (dried off immediately after calving) are combined. A decrease in placental PPIA $(P<0.001)$ and $S L C 2 A 1(P<0.001)$ and an increase in $S L C 2 A 4(P$ $<0.01$ ) occurred from d 28 to 42 of pregnancy.

fects of lactation on the development of the pregnancy. Cows in this trial were at a similar stage postpartum but were either lactating or not lactating after calving. The treatment caused large differences in hormone and metabolite concentrations, most of which were reported in a previous publication (Green et al., 2012). We observed an effect of lactation on fetal and placenta weight because the tissues weighed less when they were collected from lactating compared with nonlactating cows (Green et al., 2012). The data presented herein specifically address differences in blood glucose and insulin for lactating and nonlactating cows at the time of tissue collection and glucose and fructose concentrations within placental fluids.

Tissues from the developing pregnancy use glucose as a substrate for growth and metabolic fuel (Battaglia and Meschia, 1978). Glucose concentrations in placental fluids were approximately $40 \mathrm{mg} / \mathrm{dL}$ less than glucose concentrations in the maternal circulation (Figure 1). The lower blood glucose in lactating cows was associated with lesser glucose concentrations in placental fluids. The lower glucose concentrations in placental fluids was found on each slaughter day, with the greatest difference in lactating compared with nonlactating cows being observed on d 42 of pregnancy. By multiplying the glucose concentration by placental fluid volume, we found that the total amount of glucose in the placental fluids was greater in nonlactating cows, and differences between lactating and nonlactating cows appeared to become greater as the pregnancy progressed.
Maternal concentrations of glucose appear to affect the amount of glucose reaching the fetus and placenta (assessed by measuring the concentration of glucose in the placental fluids). This observation agrees with the general notion that glucose transport to the fetus primarily depends on SLC2A1 and SLC2A3 (Glut 1 and Glut 3 transporters; Wooding et al., 2005). Within placental tissue, glucose moves down its concentration gradient by using facilitative diffusion. The diffusion depends on the transmembrane channels formed by the facilitative glucose transporters SLC2A1and SLC2A3 (Wooding et al., 2005). Lower maternal glucose leads to lower fetal glucose because the fetus and placenta cannot sequester glucose against its concentration gradient. Furthermore, the fetus and placenta have limited capacity for gluconeogenesis (Battaglia and Meschia, 1978). The reduction in glucose reaching the pregnancy could affect how the pregnancy develops because the pregnancy depends on glucose as a substrate for tissue synthesis and metabolic energy (Battaglia and Meschia, 1978). In pregnant mice with low maternal glucose, the placenta undergoes adaptations for nutrient transport that maintain fetal growth (Coan et al., 2010). The bovine placenta however, may not have the capacity to adapt to low maternal glucose, or the capacity for placental adaptation may not occur until later in pregnancy.

An appropriate rate of development and associated consequences for slow development are poorly defined for pregnancies in cattle. Insufficient glucose reaching the placenta may cause slower placental development and a predisposition for the lactating cow to experience fetal loss. Insufficient placental development has been implicated in fetal loss before (Whitlock and Maxwell, 2008). Blood concentrations of pregnancy-associated glycoproteins (PAG), for example, were lower in cows that had fetal loss (Gábor et al., 2007; Thompson et al., 2010). Whether or not the lower blood PAG are indicative of a poorly developed placenta and whether the poorly developed placenta is a consequence of lower blood glucose are questions that will need to be addressed in future studies.

We also measured the glucose metabolite, fructose. Although the conceptus can synthesize fructose from glucose, it cannot readily metabolize fructose (Hay, 1995). Total placental fluid fructose was similar to glucose in that lactating cows had less total fructose compared with nonlactating cows.

The correlation between maternal blood glucose concentrations and placental fluid glucose concentrations in the present study (Figure 1) is consistent with facilitative glucose transport via SLC2A1and SLC2A3. Indeed, we found that SLC2A1 and SLC2A3 were highly 
expressed in placenta, whereas SLC2A1 and SLC2A3 expression in other tissues (liver and endometrium) was extremely low. Both liver and placenta expressed $S L C 2 A 4$, and expression of SLC2A4 was approximately 3 -fold greater in liver than in placenta. The relatively high expression of SLC2A4 (Glut 4 insulin-dependent glucose transporter) mRNA in placenta was an unexpected finding. An additional unexpected finding was that SLC2A4 mRNA increased from d 28 to 42 of pregnancy (Figure 3). The increase in placental SLC2A4 mRNA from d 28 to 42 was opposite to the change that we observed in SLC2A1 mRNA in placenta (which decreased from d 28 to 42). Relative changes in glucose transporter mRNA may reflect the development of cotyledons from d 28 to 42 . Our housekeeping gene control (PPIA), for example, underwent a decrease during pregnancy as well, suggesting a change in the composition of placental tissue. The decrease in $S L$ C2A1 and increase in SLC2A4 may also reflect global (placental-wide) changes in glucose transporter expression. A more complete cytological analysis of glucose transporter expression (both mRNA and protein) in placenta should be a component of future studies.

The increase in placental insulin-dependent SLC2A4 mRNA from d 28 to 42 is particularly relevant to the postpartum dairy cow that has low insulin and low glucose during lactation. If the shift in SLC2A4 expression represents a functional shift toward insulin dependence by the placenta, then the placenta would be disadvantaged in a lactating cow with low insulin. The stepwise regression that we performed eliminated all terms except day and maternal plasma insulin concentration. Subsequent regression analyses demonstrated an interaction of day of pregnancy with maternal plasma insulin concentrations (Figure 2). For later stage pregnancies (d 35 and 42), fetal and placental weight increased with increasing maternal plasma insulin concentrations. The observed relationship implies that maternal insulin concentrations may affect fetal and placental development, particularly in later stage pregnancies (d 35 or 42). The relationship with insulin for later stage pregnancies coincides with the greater SLC2A4 expression in the d 35 and 42 placenta. The association that we observed may not represent cause and effect, and further analyses will be needed to assess changes in glucose transporter expression in the placenta and their influence on the development of the fetus and maintenance of pregnancy.

One limitation of this study was that the nonlactating cows had greater BW and BCS than the lactating cows (Green et al., 2012). The nonlactating cows also had greater blood concentrations of insulin and IGF1 and lesser concentrations of growth hormone and NEFA (Green et al., 2012). The greater BW and BCS and the hormonal and metabolic differences can be explained by the experimental design that we employed. In our design, lactating and nonlactating cows were housed together in the same pen and fed the same diet so that pen and diet were not confounded with treatment (lactating versus nonlactating). Some of the responses that we observed could be explained by mechanisms that are not dependent on fetal and maternal glucose concentrations. For example, greater plasma concentrations of insulin and IGF1 in nonlactating cows may have direct effects on the pregnancy through insulin and IGF1 growth factor activity (Velazquez, et al., 2008). The greater postpartum NEFA in lactating cows may have imprinted the reproductive tract as well (Leroy et al., 2008). A caveat of this work, therefore, is the possibility that some of the effects that we observed were a consequence of the unique metabolic profile in nonlactating cows and not a consequence of the low glucose caused by lactation.

In conclusion, compared with nonlactating cows, lactating cows had fetuses and placentas that weighed less when these tissues were collected on d 28, 35, and 42 of pregnancy. Lower blood glucose in lactating cows was associated with less glucose and fructose in placental fluids compared with that of nonlactating cows. The relationship between blood and fetal glucose concentrations appeared to be explained by facilitative glucose transporters (SLC2A1and SLC2A3) that were expressed in the placenta. The insulin-dependent SLC2A4 transporter was also expressed in the placenta, and this transporter had greater expression in the older pregnancies (d 42). Lower blood glucose combined with lower blood insulin in lactating cows may lead to slower fetal development during lactation. The slower fetal development may predispose lactating cows to fetal loss if developmental milestones are not reached.

\section{ACKNOWLEDGMENTS}

This research was supported by the University of Missouri Experiment Station and Pfizer Animal Health (Kalamazoo, MI). The authors thank members of the Division of Animal Sciences at the University of Missouri, including A. Perretta, A. Brauch, C. Okamura, J. Denbigh, E. Adkins, and R. Disselhorst.

\section{REFERENCES}

Battaglia, F. C., and G. Meschia. 1978. Principal substrates of fetal metabolism. Physiol. Rev. 58:499-527.

Bauman, D. E., and W. B. Currie. 1980. Partitioning of nutrients during pregnancy and lactation: A review of mechanisms involving homeostasis and homeorhesis. J. Dairy Sci. 63:1514-1529.

Coan, P. M., O. R. Vaughan, Y. Sekita, S. L. Finn, G. J. Burton, M. Constancia, and A. L. Fowden. 2010. Adaptations in placental 
phenotype support fetal growth during undernutrition of pregnant mice. J. Physiol. 588:527-538.

De Vries, A. 2006. Economic value of pregnancy in dairy cattle. J. Dairy Sci. 89:3876-3885.

Diskin, M. G., and D. G. Morris. 2008. Embryonic and early foetal losses in cattle and other ruminants. Reprod. Domest. Anim. 43(Suppl. 2):260-267.

Frolova, A. I., and K. H. Moley. 2011. Glucose transporters in the uterus: An analysis of tissue distribution and proposed physiological roles. Reproduction 142:211-220.

Gábor, G., F. Tóth, L. Ozsvári, Z. Abonyi-Tóth, and R. G. Sasser. 2007. Early detection of pregnancy and embryonic loss in dairy cattle by ELISA tests. Reprod. Domest. Anim. 42:633-636.

Green, J. C., J. P. Meyer, A. M. Williams, E. M. Newsom, D. H. Keisler, and M. C. Lucy. 2012. Pregnancy development from d 28 to 42 of gestation in postpartum Holstein cows that were either milked (lactating) or not milked (not lactating) after calving. Reproduction 143:699-711.

Hay, W. W. 1995. Regulation of placental metabolism by glucose supply. Reprod. Fertil. Dev. 7:365-375.

Hay, W. W. 2006. Placental-fetal glucose exchange and fetal glucose metabolism. Trans. Am. Clin. Climatol. Assoc. 117:321-339.

King, G. J., B. A. Atkinson, and H. A. Robertson. 1979. Development of the bovine placentome during the second month of gestation. J. Reprod. Fertil. 55:173-180.

King, G. J., B. A. Atkinson, and H. A. Robertson. 1980. Development of the bovine placentome from days 20 to 29 of gestation. J. Reprod. Fertil. 59:95-100.

Kolath, W. H., M. S. Kerley, J. W. Golden, and D. H. Keisler. 2006. The relationship between mitochondrial function and residual feed intake in Angus steers. J. Anim. Sci. 84:861-865.

Leroy, J. L., A. Van Soom, G. Opsomer, I. G. Goovaerts, and P. E. Bols. 2008. Reduced fertility in high-yielding dairy cows: Are the oocyte and embryo in danger? Part II. Mechanisms linking nutrition and reduced oocyte and embryo quality in high high-yielding dairy cows. Reprod. Domest. Anim. 43:623-632.

Lucy, M. C. 2004. Mechanisms linking the somatotropic axis with insulin: Lessons from the postpartum dairy cow. Proc. N.Z. Soc. Anim. Prod. 64:19-23.

Rhoads, M. L., J. P. Meyer, W. R. Lamberson, D. H. Keisler, and M. C. Lucy. 2008. Uterine and hepatic gene expression in relation to days postpartum, estrus, and pregnancy in postpartum dairy cows. J. Dairy Sci. 91:140-150.

Santos, J. E., W. W. Thatcher, R. C. Chebel, R. L. Cerri, and K. N. Galvão. 2004. The effect of embryonic death rates in cattle on the efficacy of estrus synchronization programs. Anim. Reprod. Sci. 82-83:513-535.

Thompson, I. M., R. L. A. Cerri, I. H. Kim, J. A. Green, J. E. P. Santos, and W. W. Thatcher. 2010. Effects of resynchronization programs on pregnancy per artificial insemination, progesterone, and pregnancy-associated glycoproteins in plasma of lactating dairy cows. J. Dairy Sci. 93:4006-4018.

Velazquez, M. A., L. J. Spicer, and D. C. Wathes. 2008. The role of endocrine insulin-like growth factor-I (IGF-I) in female bovine reproduction. Domest. Anim. Endocrinol. 35:325-342.

Walsh, S. W., E. J. Williams, and A. C. Evans. 2011. A review of the causes of poor fertility in high milk producing dairy cows. Anim. Reprod. Sci. 123:127-138.

Whitlock, B. K., and H. S. Maxwell. 2008. Pregnancy-associated glycoproteins and pregnancy wastage in cattle. Theriogenology 70:550-559.

Wooding, F. B., A. L. Fowden, A. W. Bell, R. A. Ehrhardt, S. W. Limesand, and W. W. Hay. 2005. Localisation of glucose transport in the ruminant placenta: Implications for sequential use of transporter isoforms. Placenta 26:626-640. 